\title{
Dynamic Reservation TDMA Protocol for Wireless ATM Networks
}

\author{
Jean-François Frigon, Student Member, IEEE, Victor C. M. Leung, Senior Member, IEEE, and \\ Henry Chan Bun Chan, Member, IEEE
}

\begin{abstract}
A dynamic reservation time division multiple access (DR-TDMA) control protocol that extends the capabilities of asynchronous transfer mode (ATM) networks over the wireless channel is proposed in this paper. DR-TDMA combines the advantages of distributed access and centralized control for transporting constant bit rate (CBR), variable bit rate (VBR), and available bit rate (ABR) traffic efficiently over a wireless channel. The contention slots access for reservation requests is governed by the framed pseudo-Bayesian priority (FPBP) Aloha protocol that provides different access priorities to the control packets in order to improve the quality-of-service $(\mathrm{QoS})$ offered to time sensitive connections. DR-TDMA also features a novel integrated resource allocation algorithm that efficiently schedules terminals' reserved access to the wireless ATM channel by considering their requested bandwidth and QoS. Integration of CBR, voice, VBR, data, and control traffic over the wireless ATM channel using the proposed DR-TDMA protocol is considered in this paper. Simulation results are presented to show that the protocol respects the required QoS of each traffic category while providing a highly efficient utilization of approximately $96 \%$ for the wireless ATM channel.
\end{abstract}

Index Terms-MAC protocols, performance evaluation, wireless ATM.

\section{INTRODUCTION}

$\mathbf{I}$ $\mathrm{N}$ RECENT years, we have seen a rapid proliferation of high performance portable computers, and an increasing popularity of multimedia applications. We can therefore expect an emerging demand to connect these devices to the fixed network to transfer voice, video, data, and multimedia traffic. The wireless network will be required to be compatible with the wired broad-band communication network. Asynchronous transfer mode (ATM) was recommended by ITU-T to be the transfer protocol of the emerging broadband integrated services digital network (B-ISDN) [1] and the concept of wireless ATM (WATM) was introduced to extend the capabilities of ATM over the wireless channel [2].

\footnotetext{
Manuscript received May 3, 1999; revised September 22, 2000. This work was supported by a postgraduate scholarship and by the Natural Sciences and Engineering Research Council of Canada under Grant OGP0044286. The work of H. C. B. Chan was supported by a Hong Kong Polytechnic University Research Grant PolyU5088/99E (account code G-T041). This work is based on a paper presented at ICC'99, Vancouver, BC.

J.-F. Frigon is with the Department of Electrical Engineering, University of California, Los Angeles, CA 90095 USA (email: jeanf@ee.ucla.edu).

V. C. M. Leung is with the Department of Electrical and Computer Engineering, The University of British Columbia, Vancouver, BC, Canada, V6T 1Z4 (e-mail: vleung@ece.ubc.ca).

H. C. B. Chan is with the Department of Computing, Hong Kong Polytechnic University, Hong Kong, China (email: cshchan@comp.polyu.hk).

Publisher Item Identifier S 0733-8716(01)00829-0.
}

A major issue related to the realization of WATM networks is the selection of a medium access control (MAC) protocol that will efficiently and equitably allocate the scarce and valuable radio medium among the competing mobile stations while respecting the quality-of-service (QoS) requirements of each admitted connection. For compatibility with the wired ATM network, the WATM MAC protocol must support the standard ATM service classes: constant bit rate (CBR), variable bit rate (VBR), and available bit rate (ABR) traffic. In the past years, several projects have developed MAC protocols for wireless ATM networks [3]-[10], and most of these protocols are designed to support multimedia ATM traffic. Among the known allocation algorithms for WATM, we can underline the protocols presented in [5], [8]-[10] as being the most complete and efficient. In [5] and [10], the proposed allocation algorithms require an explicit transmission of the current requirement of each active connection from the mobile to the base station. This produces a large overhead and results in a throughput reduction. In this paper, we use a novel predictive approach where the base station estimates from a limited number of parameters the current requirements of the connections. A similar predictive approach has been proposed in [9] for VBR traffic. However, with the method that we are proposing in this paper, by forcing the VBR source to be rate-controlled, we are able to significantly increase the performance. Our method also employs a cell control algorithm that complies with the ATM Forum specifications, which has not been accomplished before for the wireless environment. Another area where most of the previously proposed protocols present some pitfalls is related to the random access protocols employed for initial access. They either limit the delay-throughput performance [5], [8], [9] or restrict the number of users [10]. Generally they do not offer access priorities between different service classes. In the MAC protocol presented in this paper, we adopt a new random access protocol that allows us to increase the throughput performance while maintaining the required QoS, by enabling appropriate control traffic access priorities for different service classes.

This paper describes the dynamic reservation TDMA (DR-TDMA) MAC protocol that is capable of maintaining a high channel utilization while respecting the QoS requirement of each connection depending on its ATM traffic class. To improve the performance of the protocol, DR-TDMA uses the novel framed pseudo-Bayesian priority (FPBP) Aloha algorithm [11] to manage the access to the control minislots such that contention can be minimized and access priorities can be provided to control packets in order to improve the QoS offered to connections with time sensitive traffic. Moreover, 


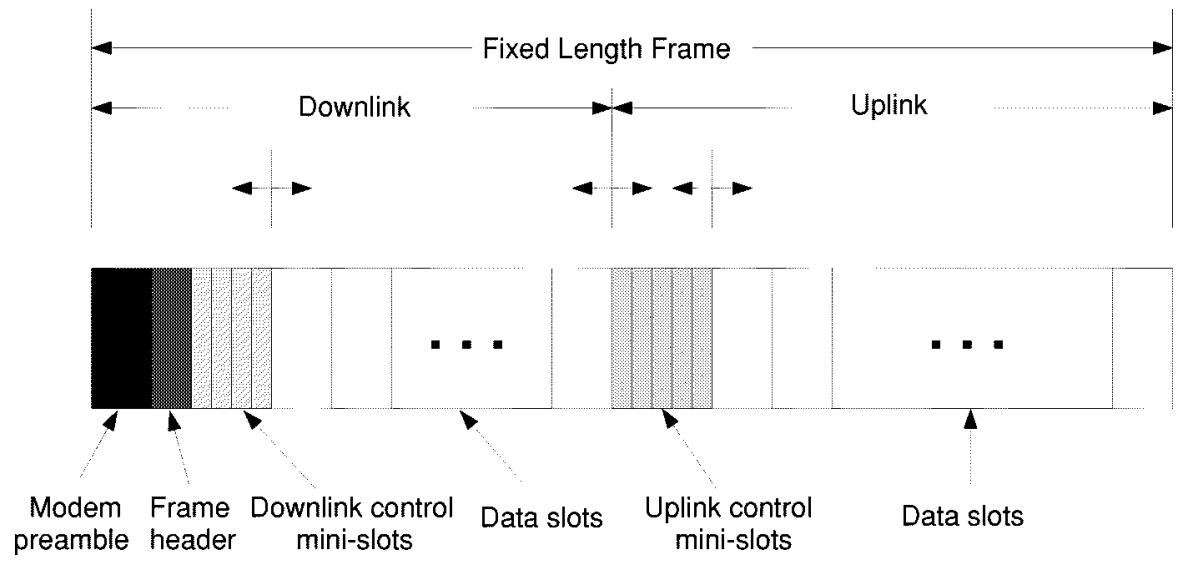

Fig. 1. DR-TDMA frame structure.

the FPBP protocol dynamically adjusts the number of uplink (mobile to base station) control slots as a function of the traffic load to optimize the channel utilization. A key feature of our DR-TDMA protocol is the bandwidth allocation algorithms used to distribute the bandwidth among the CBR, voice, VBR, data, and control traffic. These include, in particular, an efficient rate-based allocation algorithm for VBR traffic, which incorporates a cell control algorithm to enforce the conformance of a VBR flow with the connection's declared traffic parameters (sustained cell rate, peak cell rate, and burst tolerance). A method to integrate the different allocation algorithms to provide ubiquitous wireless ATM services is described. We present simulation results to illustrate the performance of the DR-TDMA protocol under a wide range of traffic conditions. These results show that the protocol proposed in this paper outperforms, under similar traffic conditions, previous protocols.

The paper is organized as follows. Section II gives an overview of the DR-TDMA protocol. In Section III, the source models are presented. In Section IV, we describe the resource allocation algorithms. An evaluation of DR-TDMA performance by simulations is presented in Section V. Finally, Section VI concludes the paper.

\section{DYNAMIC RESERVATION TDMA PROTOCOL}

\section{A. Frame Structure}

A DR-TDMA protocol is adopted to multiplex multimedia ATM connections over a single time division duplex (TDD) radio channel. Fig. 1 illustrates the frame structure employed by the DR-TDMA protocol. The fixed length DR-TDMA frame is time-duplexed into an uplink and downlink channel and the boundary between these two parts is dynamically adjusted as a function of the traffic load. The downlink and uplink channels are dynamically divided into control and data transmission periods, each consisting of an integer number of slots where each slot has the same length as a data packet. Slots assigned for control purposes are further divided into three control minislots, each carrying a control packet. The base station has absolute control in determining the number of each type of slots during
TABLE I

DR-TDMA MAC FRAME PARAMETERS

\begin{tabular}{l|r}
\hline Channel bit rate & $8.528 \mathrm{Mbps}$ \\
Frame duration & $2 \mathrm{~ms}$ \\
Uplink data slot size & 60 bytes \\
Uplink control slot size & 20 bytes \\
Preamble size & 16 bytes \\
Frame header & 16 bytes \\
Number of slots per frame & 35 \\
Number of control mini-slots per slot & 3 \\
\hline
\end{tabular}

a frame period and which mobile stations will receive or send information using the data slots. System parameters adopted for the DR-TDMA protocol are according to the literature [2], [9] and are summarized in Table I. It should be noted that the only parameters that directly influence the MAC protocol efficiency are: the frame duration, the number of control and data slots per frame, and the ratio of data slot size to control slot size.

\section{B. Protocol Mechanisms}

In the uplink channel, control slots provide a communication mechanism for a mobile station to send a reservation request during the contention phase of the connection, while the data slots supply it with contention-free bandwidth resources during the data transmission phase. An uplink control packet is sent whenever a mobile station needs to signal the base station and it does not have an assigned data slot. Uplink control packets provide the base station with the traffic characteristics and source status of the corresponding mobile stations'connections. Uplink control packets are sent in contention-mode according to the FPBP protocol described in Section II.C. This protocol assigns contention access priorities to mobile stations according to the required QoS of their connections. The contention delays of time sensitive control packets are thus reduced. Feedback for the uplink control packets is sent in the downlink control packets in the next frame. Mobile stations which control packets have experienced a collision will repeat the contention process in subsequent frames until they are successful. 
After completing the contention procedure, the mobile station can use the data slots assigned by the base station without undergoing further contentions. While a mobile station has access to reserved data slots, traffic parameters and status information, e.g., specifying requirements for additional data slots, can be sent piggybacking on the data packets. The base station will use these traffic parameters to allocate uplink data slots to each reserving station according to the allocation algorithms presented in Section IV. When a connection has successfully sent its request, it enters the data transmission phase and monitors downlink control slots in each subsequent frames to receive its slot assignments, which identify the uplink data slots in the current frame in which the mobile station should send its packets.

\section{Contention Access Scheme}

In a reservation MAC protocol such as DR-TDMA, before obtaining contention free access to the channel, a mobile must wait for its request packet to be successfully sent to the base station in contention with control packets from other connections. Therefore, the contention phase that a connection goes through before obtaining contention-free packet transmissions has a major effect on the delay QoS of a reservation MAC protocol. As the QoS of some classes of traffic is more sensitive to the contention delay than others, this calls for the design of a contention access scheme that provides a lower contention delay to time sensitive control packets while being adapted to the structure of DR-TDMA. The FPBP protocol described in detail in [11] has been designed to satisfy these requirements, and is employed by mobile stations to access uplink control mini-slots. This protocol is an extension of Rivet's pseudo-Bayesian Aloha stabilization algorithm [12] that provides multiple levels of access priorities and is adapted to the frame structure of DR-TDMA.

We now summarize the control traffic access algorithm. Suppose that $K^{t}$ control mini-slots are available in frame $t . K^{t}$ is computed each frame using the algorithm presented in Section IV-D. There are $p$ different control priority classes with arrival intensities $\lambda_{1}, \ldots, \lambda_{p} . \lambda_{i}$ is the average number of control packet arrivals per frame of priority class $i$ from all mobile stations and is computed using a moving time-average of the number of successful uplink control packet transmissions from class $i$ per frame. A lower index corresponds to a higher priority class for the control packets.

Let $n_{n c}$ be the number of idle control slots or control slots with successful access, and $n_{c}$ the number of control slots with collisions in frame $t-1$. At the beginning of each frame $t$, for each priority class $i$, an estimate $\hat{n}_{i}^{t}$ of the number of waiting control packets is updated from $\hat{n}_{i}^{t-1}, \hat{\gamma}_{i}^{t-1}$ (the effective priority parameter), $K^{t-1}$ and the feedback $\left(n_{n c}\right.$ and $\left.n_{c}\right)$ for frame $t-1$ according to the rule [11]:

$$
\begin{aligned}
\hat{n}_{i}^{t}= & \lambda_{i}+n_{n c} \max \left(0, \frac{\hat{n}_{i}^{t-1}}{K^{t-1}}-\hat{\gamma}_{i}^{t-1}\right) \\
& +n_{c}\left(\frac{\hat{n}_{i}^{t-1}}{K^{t-1}}+\frac{\hat{\gamma}_{i}^{t-1}}{e-2}\right) .
\end{aligned}
$$

The effective priority parameter $\hat{\gamma}_{i}^{t}$ of each class is then computed using the following prorating algorithm [11]: for (each priority class $i$ )

$$
\begin{aligned}
& \hat{\gamma}_{i}^{t}=\min \left(\hat{n}_{i}^{t} / K^{t}, 1-\sum_{j=1}^{i-1} \hat{\gamma}_{j}^{t}\right) \\
& L=1-\sum_{i=1}^{p} \hat{\gamma}_{i}^{t}
\end{aligned}
$$

for (each priority class $i$ )

$$
\hat{\gamma}_{i}^{t}=\hat{\gamma}_{i}^{t}+\frac{\lambda_{i}}{\sum_{j=1}^{p} \lambda_{j}} L .
$$

Each waiting control packet is then independently transmitted in the uplink control period of frame $t$ according to the transmission probability $q_{i}^{t}$ of the priority class $i$ it belongs to. Transmission probabilities are computed centrally in the base station as follows:

$$
q_{i}^{t}=\min \left(1, \frac{\hat{\gamma}_{i}^{t}}{\hat{n}_{i}^{t}} K^{t}\right)
$$

If a control packet is transmitted in a given frame, one of the available uplink control minislots is independently chosen with equal probability for its transmission. Using this algorithm, the control traffic throughput is maintained closed to its optimum value of $e^{-1}$ while high priority control packets enjoy a higher access probability.

\section{SOURCE MODELS}

The source models presented in this section are not only useful for the performance analysis of the DR-TDMA protocol; the resource allocation algorithm proposed in Section IV is also partially based on the selected source models. The voice and data source models do not require any traffic policing from the transmitting mobile stations. On the other hand, traffic shaping is mandatory for the VBR sources, and allows the implementation of an efficient VBR allocation algorithm which meets the QoS specifications defined by the ATM Forum. The cell control algorithm is similar to what would be required from a VBR source connected to an ATM network, and the source rate control is in conformance with the structure of a typical video coder such as MPEG.

\section{A. Voice Source Model}

A voice source generates a signal that follows a pattern of talkspurts separated by silent gaps. A speech activity detector can be used to detect this pattern. Voice packets (cells) are thus transmitted only during periods of speech activity to reduce the traffic and enable statistical multiplexing. Therefore, a voice source can be described by an ON/OFF model: the source alternates between the ON state where the source generates packets at rate $R_{v}$, the encoder bit rate, and the OFF state where no packets are generated. Durations of ON and OFF states are modeled by 
TABLE II

Voice SOURCE Model PARAMETERS

\begin{tabular}{l|r}
\hline$t_{b}^{v o}$ & $1.00 \mathrm{~s}$ \\
$t_{i}^{v o}$ & $1.35 \mathrm{~s}$ \\
Activity ratio & 0.426 \\
$R_{v}$ & $24 \mathrm{Kbps}$ \\
$T_{v}$ & $16 \mathrm{~ms}$ \\
MTD & $16 \mathrm{~ms}$ \\
Priority & high \\
\hline
\end{tabular}

TABLE III

DATA CONNECTION PARAMETERS

\begin{tabular}{c|c}
\hline Data. source bit rate & $19.2 \mathrm{Kbps}$ \\
\hline Average interarrival time between data burst $\left(t_{d}\right)$ & $100 \mathrm{~ms}$ \\
\hline Beta parameter of the gamma function $(\beta)$ & 3 \\
\hline Mean burst length $\left(\mu_{b}\right)$ & 5 cells \\
\hline Maximum transfer delay (MTD) & $60000 \mathrm{~ms}$ \\
\hline Priority & low \\
\hline
\end{tabular}

exponential distributions with mean values $t_{b}$ and $t_{i}$, respectively. This model is similar to the "slow" speech activity detector model described in [13], and we have therefore selected the same values for parameters $t_{b}$ and $t_{i}$. A voice source is also characterized by the packetization delay to assemble a packet payload $T_{v}$. If a voice packet is not sent within its maximum transfer delay (MTD), it should be dropped. In our voice source model, the MTD is set equal to the packetization delay $T_{v}$ (i.e., one voice packet is buffered). Table II summarizes the voice source model parameters.

\section{B. Data Source Model}

Data sources are represented by a model in which batches of packets (ATM cells) arrive in the mobile station buffer at a certain rate. This model is in accordance with the ATM adaptation layer AAL5 used for ABR and UBR data traffic, which segments packets received from the upper layer into 48-byte ATM cells. It is reasonable to assume that these cells arrive in the data buffer of a mobile station at approximately the same time.

The interarrival time between groups of packets is assumed to be exponentially distributed with mean $t_{d}$. The number of packets in each batch arrival is given by $n=\lceil x\rceil$, where $x$ is gamma distributed with parameters $\beta$ and $\theta$. Using a random number generator, we find that $n$ has a mean $\mu_{b}$ approximately given by $1 / \theta+0.5$ and a mode value of $(\beta-1 / \beta \theta)+0.5$. We have selected this model instead of the usual geometric model because the latter one has a mode value of one which is not realistic for ATM data traffic. The data parameters used in this paper are presented in Table III.

\section{VBR Source Model}

A VBR source generates a traffic flow at a rate that varies with time. If the transmission rate varies continuously in an uncontrolled manner, it would require much control traffic from the mobile station to keep the base station updated about the buffer state in the VBR source. Fortunately, real VBR sources such as MPEG video encoders do not generate traffic flows which continuously vary with time. Instead, their bit rates typically vary among a fixed number of possible rates [14], [15]. Also, a general VBR traffic flow can be rate-controlled using such algorithms as presented in [16]. We therefore consider a discrete rate VBR model that gives the basis for efficient slot scheduling with a minimal amount of control traffic. In this model, the source traffic flow when cell $c$ arrives has an instantaneous cell rate $R_{c}=l^{c} R_{b}$, where $R_{b}$ is the basic source cell rate. After the arrival of cell $c$, the cell arrival rate changes to the value $R_{c+1}=l^{c+1} R_{b}$.

The above discrete rate VBR model can be modeled mathematically by a superposition of $S$ ON/OFF sources, similarly to what was proposed in [17]. Each ON/OFF source $s(s=1, \ldots, S)$ alternates between the ON and OFF states. Durations of ON and OFF states are assumed to be exponentially distributed with means $t_{b}^{\mathrm{vbr}}$ and $t_{i}^{\mathrm{vbr}}$, respectively. Although this mathematical model is not representative of every types of VBR sources, it provides an easy means to evaluate the efficiency of the proposed protocol and demonstrate its properties. It should be stressed that this ON/OFF model is only used for performance evaluation purposes. The allocation algorithm proposed in Section IV-C is, therefore, not based on this ON/OFF model but on the general rate-controlled VBR source model described in the previous paragraph.

When a VBR connection is admitted in the WATM network, it is required to specify the parameters of its traffic flow. Cells that conform to the specified traffic contract receive a high QoS, while nonconforming cells are served on a best-effort basis without any QoS guarantee. Nonconforming cells are not necessarily discarded in order to take advantage of unused resources in the network.

When a packet (cell) arrives from the VBR rate-controlled source, the cell control algorithm shown in Fig. 2 determines if the packet conforms with the VBR traffic parameter. An arriving packet is considered to be in compliance with the specified traffic parameter if, when the packet arrives from the source, there is a token in the guaranteed token pool. In this case, a token is removed from the guaranteed token pool and the packet is tagged as a guaranteed packet and will receive priority service which guarantees a high QoS. Otherwise, the packet is tagged as a best effort packet and will receive a low QoS. Cells are queued in the output buffer until the mobile station receives a slot allocation for their transmissions over the wireless channel. Tokens arrive at a constant rate of $g R_{b}$ and are queued in the guaranteed token pool, which can store a maximum of $W$ tokens. Tokens arriving at the guaranteed token pool when it is full are lost. The above cell control algorithm is similar to the virtual scheduling algorithm considered by the ATM Forum [18]. Similarly to voice packets, VBR packets are discarded when they exceed their MTD.

\section{BANDWIDTH AllocATION AlgORITHMS}

The main objective of a WATM bandwidth allocation algorithm is to efficiently exploit statistical multiplexing while providing the negotiated QoS to each admitted connection in the 


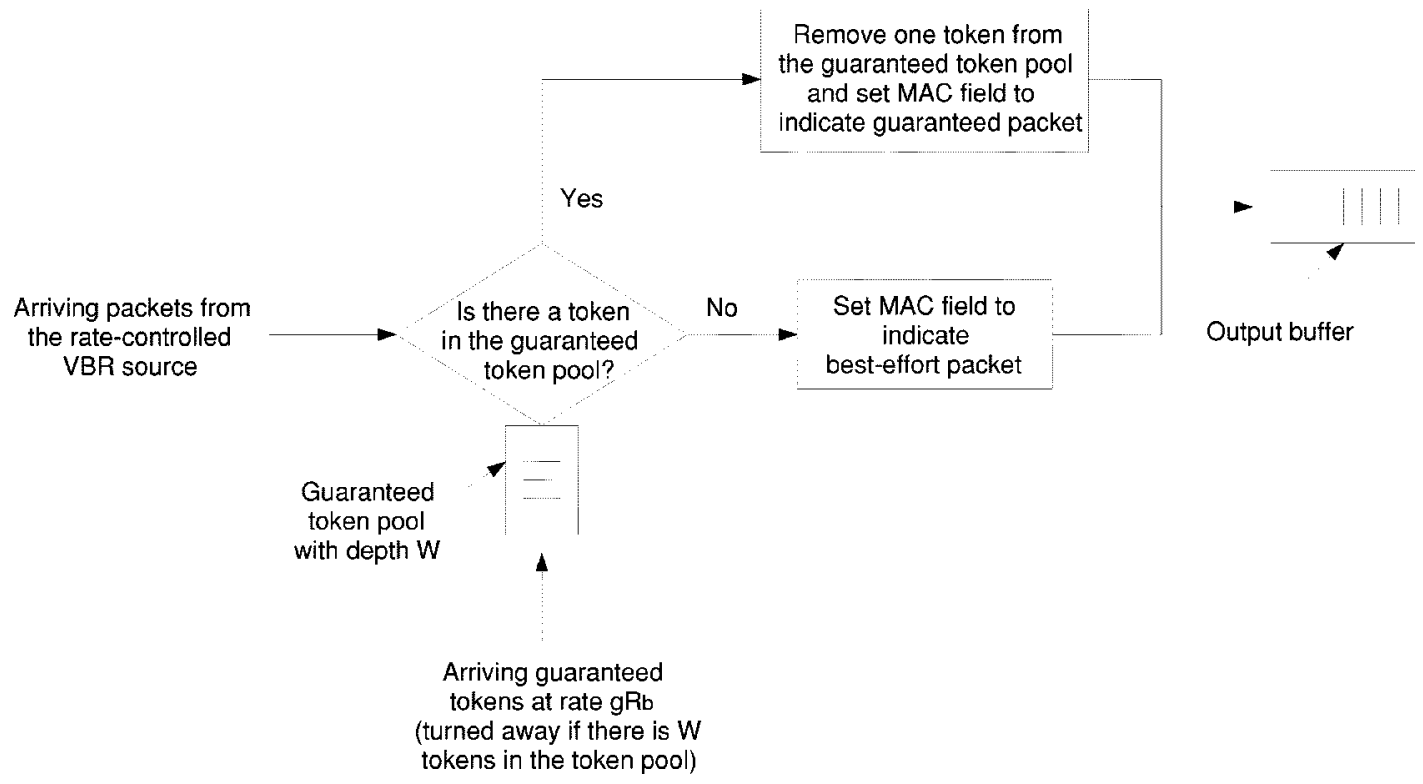

Fig. 2. Cell control algorithm.

network. In this paper, we only consider the bandwidth allocation for uplink transmissions since downlink transmissions can be scheduled in the same manner as in a wired ATM switch. For our DR-TDMA protocol, the base station scheduler decides, based on its current knowledge of the buffer states in the mobile stations and the connection traffic parameters, how to assign data slots to each connection and the number of slots to allocate for the control traffic.

\section{A. Slot Allocation Algorithm for Voice and CBR Traffic}

A CBR connection is a special case of a voice connection consisting of a single period of activity lasting for the whole connection time. We can therefore use the allocation algorithm that we will describe for voice connections to allocate slots for CBR connections. At the beginning of a talkspurt, the mobile sends a high priority control packet to the base station and the connection is then considered as active. For each active connection, the scheduler predicts the packet arrivals using the declared constant bit rate provided in the initial control packet. The slot allocation algorithm for voice traffic uses a time-to-expiry (TTE) approach in which the connection which voice packet will be discarded first receives the next slot allocation [19]. At the end of a talkspurt, the mobile sets a flag in the last voice packet to indicate to the scheduler that the connection is no longer active.

\section{B. Slot Allocation Algorithm for Data Traffic}

For the data traffic allocation algorithm, the base station records the buffer length status of each connection using the control information transmitted by the mobile. When a batch of packets arrive at a mobile, it sends the number of packets in the new batch arrival either piggybacked to a data packet or in a low priority control packet. After the buffer length status of data connections has been updated in the base station, the scheduler allocates slots to the connections using a fair bandwidth allocation algorithm [19]. When a mobile receives its slots allocation from the scheduler in the downlink control slots, it transmits packets that are waiting in its buffer on a first-in-first-out basis.

\section{Slot Allocation Algorithm for VBR Traffic}

The rate-based VBR allocation algorithm that we propose maintains in the base station a virtual status of each admitted VBR connection and allocates slots according to the specified QoS and the virtual state of the VBR connection in the base station. Information about the current cell arrival rate of a VBR source is transmitted from the mobile station to the base station either piggybacked to a data packet or in a control packet, and is used by the scheduler to predict the buffer status of the connection (number of packets, types of packets-guaranteed and best-effort, arrival times, number of tokens in guaranteed token pool). These predictions are done by reproducing the source arrival process in the base station scheduler.

When a new VBR connection is established, the mobile station sends a high priority control packet using the FPBP protocol. This packet contains the VBR source traffic parameters. During the connection, each data packet carries a MAC field which indicates the packet type and the change in source rate level upon the packet's departure from the VBR source (i.e., for packet $c$ it is equal to $l^{c+1}-l^{c}$ ). The overhead required for the allocation algorithm in data packets is thus relatively small. One bit can indicate the packet type while the rate change can be encoded in a small number of bits. For example, if we limit the variations of the source parameter $l^{c}$ to an absolute value smaller than 15, then five bits are enough for this MAC field.

If a rate change occurs for one of the predicted packets, then subsequent predictions will be erroneous. However, when the base station subsequently receives the packet with the rate change indication, the newly received information as well as information stored with each virtual packet (current arrival rate, arrival time, guaranteed token buffer status, ...) allows the virtual buffer status prediction to be updated correctly starting from this packet. However, when the cell arrival rate is equal to zero, or a packet with a nonzero rate change field 
is dropped in the mobile station (a packet can be dropped in the mobile station due to its MTD being exceeded or because it is a best-effort packet that cannot be transmitted in sequence), the base station can no longer correctly predict cell arrivals and buffer status. In this case, the information required by the scheduler is transmitted in control packets.

At the end of each frame, the base station updates the virtual status prediction of each VBR connection based on the connection parameters and the information received during the frame either piggybacked to a data packet or directly in a control packet. Note that the scheduler only predicts arrival occurring before the beginning of the frame for which the slot allocation will be done. When the virtual status of all the connections has been updated in the base station, the scheduler allocates slots to the VBR connections according to the virtual buffer status and QoS of each connection. The slot allocation algorithm is divided into two parts: guaranteed allocation and best-effort allocation. First, the scheduler allocates the available slots to the VBR connections that have waiting guaranteed packets using the time-to-expiry algorithm. Then, if there are still available slots, the scheduler allocates the remaining slots to the VBR connections with best-effort packets in their virtual queue according to the fair bandwidth allocation algorithm.

When the allocations for guaranteed and best-effort packets have been made, the base station announces in the downlink control slots the number of slots allocated to each mobile stations. Each VBR connection will transmit the packets in the same order as they arrived in its buffer, with priority given to guaranteed packets. If, in the current frame, a best-effort packets cannot be transmitted but a guaranteed packet with a later arrival time is transmitted, then the best-effort packet is dropped to preserve packet sequencing. Technical details of the implementation of the VBR prediction algorithm are available in [19] and [20].

\section{Allocation Algorithm for Control Traffic}

The number of slots allocated to control traffic is computed using the estimate of the number of backlogged control packets determined by the FPBP algorithm $\left(\sum_{i=1}^{p} \hat{n}_{i}^{t}\right)$. Let CR be the number of control mini-slots per slot, and CSmax the maximum number of slots that can be allocated for control purposes. The goal of the allocation algorithm for control traffic is to estimate the minimum number of control slots, smaller than the maximum allowed by CSmax, such that every control packet waiting for transmission will be sent during the next frame. This allows a dynamic adjustment of the number of control slots to obtain a compromise between a small number of control slots and a low transmission delay. The number of uplink control slots requested for frame $t, r^{t}$, is determined as follows:

$$
\begin{aligned}
& \text { if }\left(\sum_{i=1}^{p} \hat{n}_{i}^{t} \geq 1\right) \\
& r^{t}=\min \left(\left\lceil\frac{\sum_{i=1}^{p} \hat{n}_{i}^{t}}{\mathrm{CR}}\right\rceil, \text { CSmax }\right)
\end{aligned}
$$

else

$$
r^{t}=0 \text {. }
$$

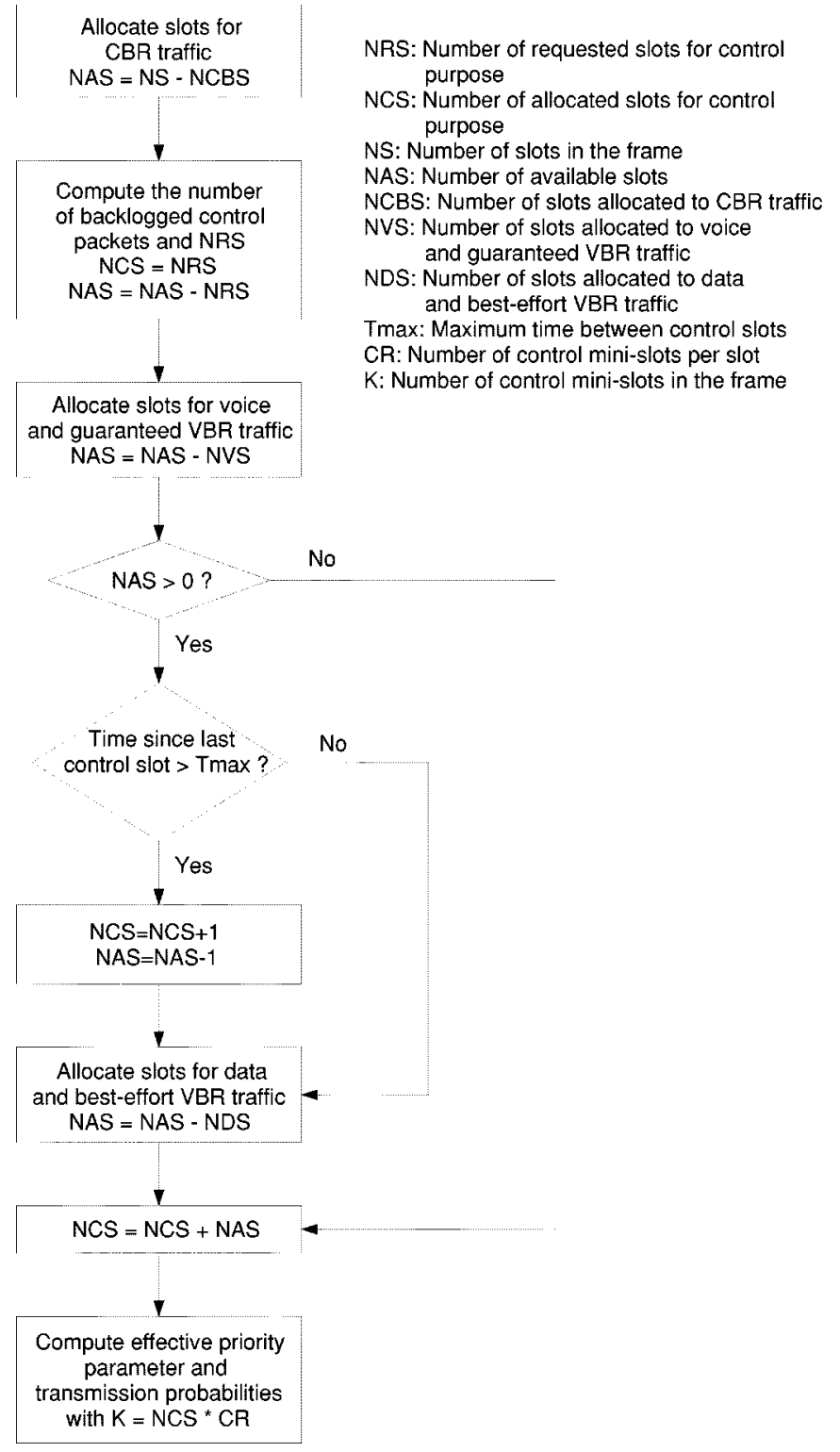

Fig. 3. Traffic integration flow chart.

Furthermore, before allocating slots for best-effort traffic, if the time since the last allocated control slot exceeds a parameter Tmax, then a control slot is allocated for the next frame. This process ensures that a contention period will be regularly available to allow potentially time sensitive control packets to be transmitted. After slot allocations for user traffic have been done, all unused slots are allocated to the contention period for control packets.

\section{E. Traffic Integration}

We have described above how slot allocations are independently managed for each type of traffic. However, since the different WATM services share the same resources, an effective interaction between the allocation algorithms is needed to maximize the utilization efficiency of the shared resources. Fig. 3 shows the algorithm used to integrate the different types of traffic for uplink transmissions using the DR-TDMA protocol. The available slots are distributed first to CBR traffic, 
second to control traffic, then to voice and VBR guaranteed traffic, and finally to data and VBR best-effort traffic. If there are any slots left over, they will be assigned to control slots. This order of allocation is based on the different priorities and QoS requirements of the ATM services.

The allocations for voice and VBR guaranteed traffic are made jointly by maintaining a single list of the active voice connections and VBR connections with queued guaranteed packets in increasing order of TTE. Connections are then allocated slots in order of increasing TTE. This allows guaranteed VBR packets and voice packets to receive the same QoS according to their traffic parameters. Similarly, the algorithm maintains a single list containing the active data connections and the VBR connections with queued best-effort packets, and the fair bandwidth allocation algorithm is executed with this list.

For control slots, the number of backlogged control packets at the beginning of the frame, and accordingly the number of required uplink control slots, is computed using the feedback from the previous frame's control slots. After the allocations for CBR, control, voice, VBR, and data traffic have been made, the number of control slots available in the current frame is known, and the transmission probabilities can then be computed. Finally, the slot allocations and the contention parameters (number of control slots and transmission probabilities) are announced in downlink control slots.

\section{Performance Evaluations}

A C++ simulator has been written to evaluate the performance of the proposed DR-TDMA protocol. The simulator exactly implements the scheduling algorithm described in Section IV. The results therefore reflect the impact of signaling delay and inaccurate instantaneous prediction on the protocol performance. In this section we only present a subset of the simulation results that have been obtained (see [19] for additional results).

To focus on the MAC performance, we consider a perfect radio channel without errors and fading, i.e., we assume properly designed signaling and channel coding schemes which minimize transmission errors. Each simulation was run for a minimum simulation time of 5000 seconds to ensure accuracy of the results. For the FPBP access protocol, the number of priority levels $p$ is set equal to 2. Voice and VBR control packets are assigned priority level 1 and data control packets are assigned priority level 2 . The length of the window used for computing the moving time average of the number of successful control packet transmissions for each traffic class is set to 100 frames. CSmax has been set to 2 and Tmax to $12 \mathrm{~ms}$. We have assumed an infinite buffer at each mobile station such that cell losses are only due to exceeding the MTD.

In order to evaluate the efficiency of the uplink MAC protocol, we assume that downlink control slots do not consume any transmission bandwidth. The throughput is defined as the ratio of the average number of slots used for data packet transmissions per frame (excluding control packets) to the total number of slots available per frame ( 35 slots). The offered load and achieved throughput thus include cell headers but do not include control packets. Therefore, control traffic contributes to a throughput reduction. We also did not consider preamble
TABLE IV

VBR CONNECTION PARAMETERS

\begin{tabular}{c|c|c}
\hline Connection number & $\# 1$ & $\# 2$ \\
\hline Connection Bit Rate & $250 \mathrm{Kbps}$ & $667 \mathrm{Kbps}$ \\
\hline Basic source cell interarrival $\left(1 / R_{b}\right)$ & $7.68 \mathrm{~ms}$ & $3.6 \mathrm{~ms}$ \\
\hline Number of ON/OFF sources $(S)$ & 15 & 25 \\
\hline Average ON state length $\left(t_{b}^{v b r}\right)$ & $100 \mathrm{~ms}$ & $100 \mathrm{~ms}$ \\
\hline Average OFF state length $\left(t_{i}^{v b r}\right)$ & $200 \mathrm{~ms}$ & $300 \mathrm{~ms}$ \\
\hline Guaranteed parameter $(g)$ & 5 & 5,10 and 25 \\
\hline Guaranteed token pool depth $(W)$ & 1 and 10 & 1 \\
\hline Maximum transfer delay $(\mathrm{MTD})$ & $50 \mathrm{~ms}$ & $50 \mathrm{~ms}$ \\
\hline
\end{tabular}

traffic sent at the connection admission, which will further reduce the achievable throughput.

\section{A. Performance With Integrated Traffic}

A first set of simulations have been run with 100 voice connections with parameters presented in Section III-A, 100 data connections characterized by the parameters in Table III, and VBR connections with parameters in Table IV (we used for this set of simulation VBR connections \#1 with $W=1$ ). The number of VBR connections is a varying parameter to illustrate the performance of the integrated system.

Fig. 4 presents the voice and VBR cell loss rates as functions of the number of VBR connections. It is apparent that the integrated allocation algorithm meets the VBR and voice QoS (i.e., $1 \%$ cell loss rate [21]) for all the traffic conditions. We note that the cell loss rate of VBR traffic is higher than for voice traffic; this is due to the fact that a portion (on average 14\%) of the VBR traffic receives a best effort service. The VBR total cell loss rate includes both guaranteed and best-effort packet losses. On the other hand, even if voice and VBR guaranteed traffic receives the same service, the voice cell loss rate is higher than that of guaranteed VBR cells because of voice cell losses caused by delayed control packets, which is not a performance determining factor for guaranteed VBR traffic. In fact, no loss of VBR guaranteed packet have been observed in the simulations. Even for a throughput of 99\%, as indicated by Fig. 5, the QoS of voice and VBR traffic is maintained at the expense of data QoS.

The flattening of the voice cell loss rate between 13 and 14 VBR connections can be explained by the fact that the performance of voice traffic is mainly determined by contention traffic, which in turn is influenced by the volume of voice and data control traffic and the number of control slots available. When the number of VBR connections increases, the number of available control slots decreases causing an increase in contention which contributes to the initial increasing cell loss rate. Whereas before the flat the voice and data control traffic is almost constant, when the number of VBR connections reaches 13 we see from the delay results in Fig. 6 that the data delay dramatically increases. At this point, the data control traffic decreases because the buffers in the corresponding mobile stations are seldom empty, such that an increasing number of requests are sent to the base station piggybacked to data packets. Therefore, the two opposing effects result in the flat. Beyond this 


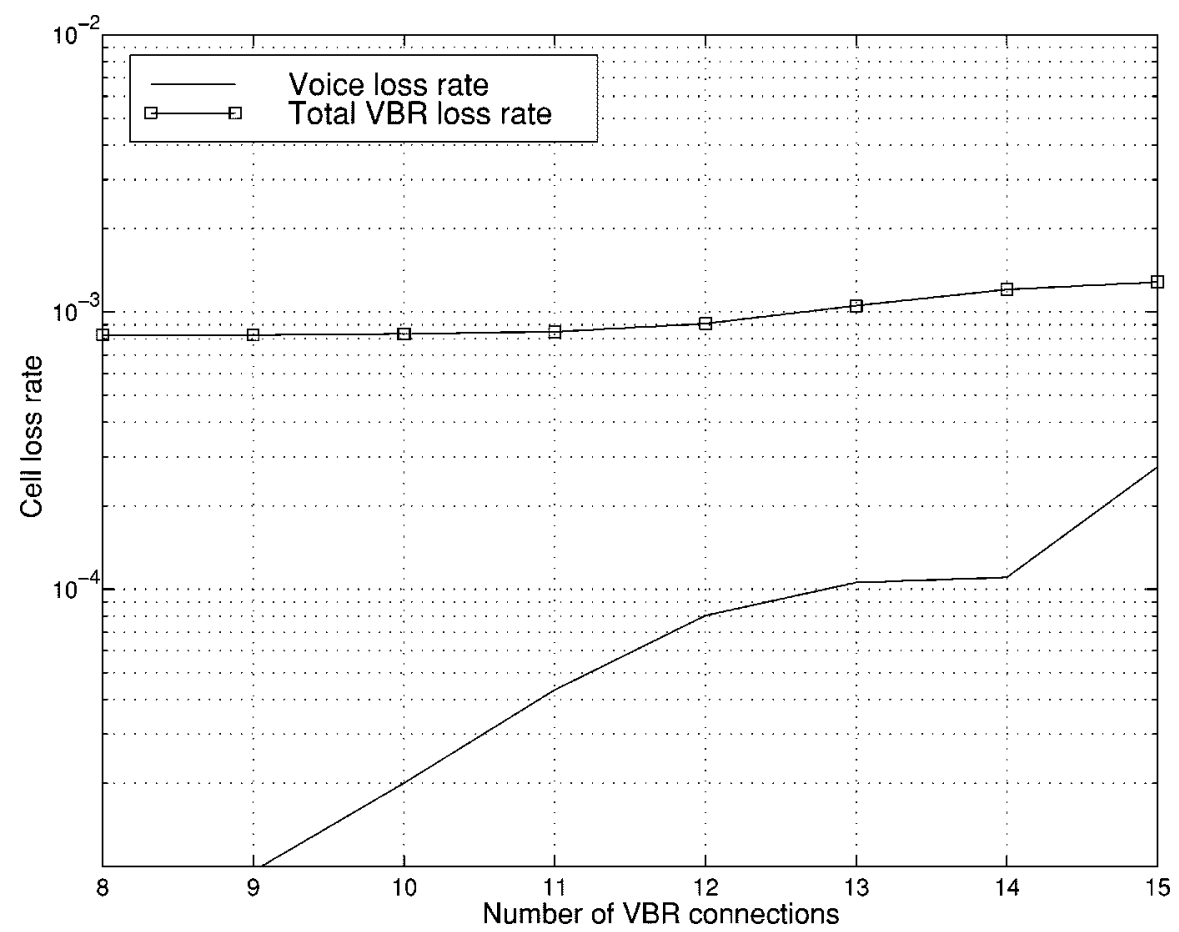

Fig. 4. Cell loss rate as a function of the number of VBR connections for the integrated system.

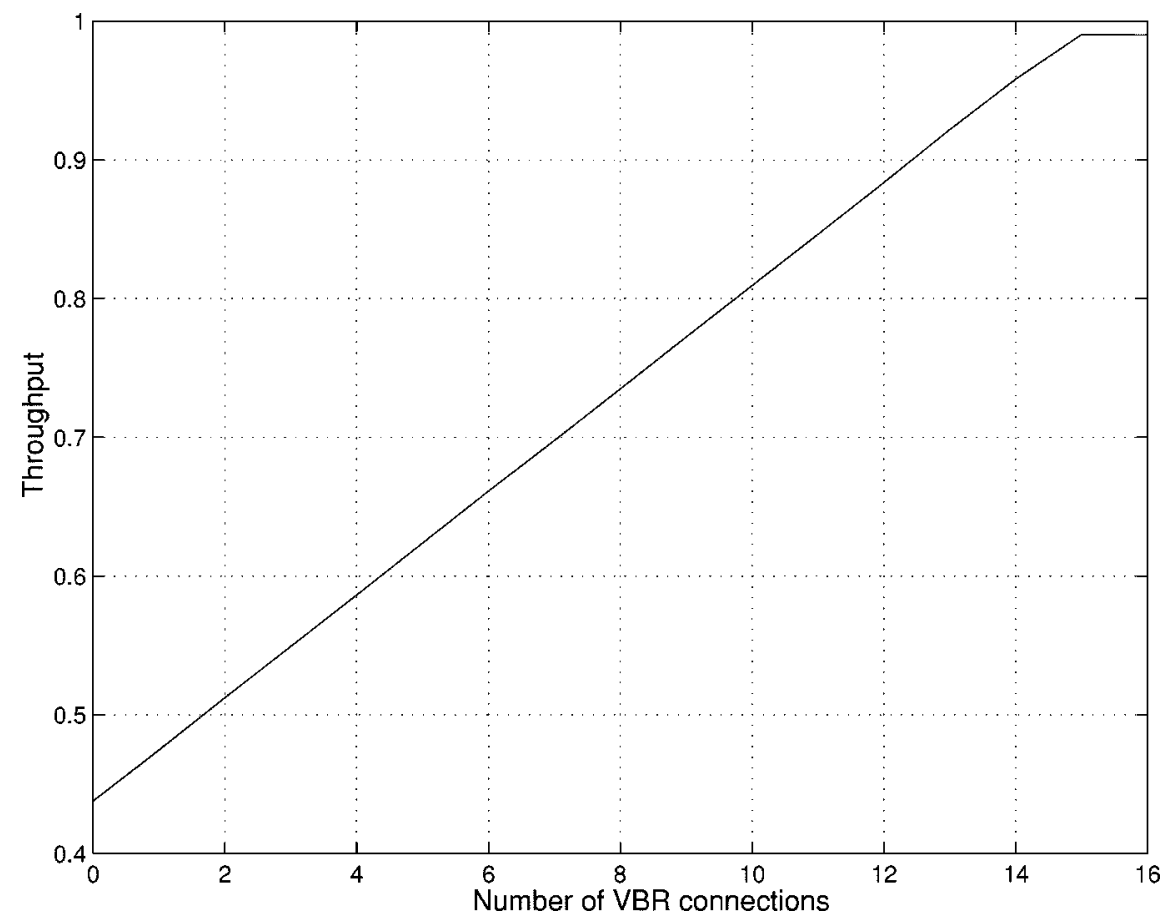

Fig. 5. Throughput as a function of the number of VBR connections for the integrated system.

point, the decreasing number of available control slots causes further increases in the voice cell loss rate.

We can observe from Fig. 6 that the minimum VBR cell delay is $2 \mathrm{~ms}$ and for data traffic there is a minimum delay of $4 \mathrm{~ms}$. These minimum delay results from the allocation algorithm since the base station does not predict future arrivals at the mobile station but arrivals that has occurred before the current frame. Therefore, VBR slot allocation in the current frame are for packets that arrived in the previous frames, which ex- plains the fact that there is a minimum transmission delay of one frame $(2 \mathrm{~ms})$. On the other hand, data traffic information about batch arrivals can only be transmitted in the frame following their arrival, therefore adding an additional one frame delay for a minimum transmission delay of two frames (4 ms).

Fig. 6 shows that the VBR cell delay remains approximately at its minimum value of $2 \mathrm{~ms}$ for all throughput values. On the other hand, data traffic experiences a minimum delay of $4 \mathrm{~ms}$ while the throughput is below $80 \%$. When the offered load in- 


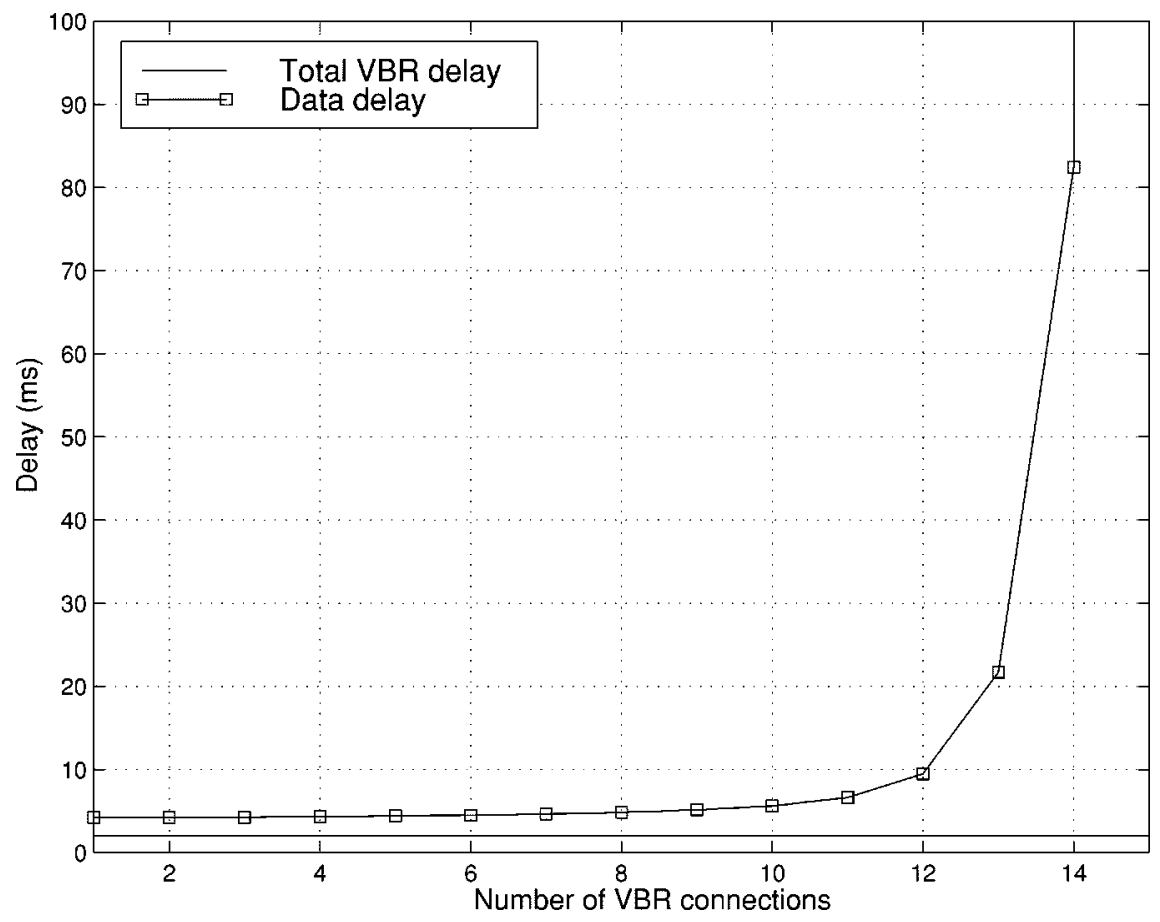

Fig. 6. Delay as a function of the number of VBR connections for the integrated system.

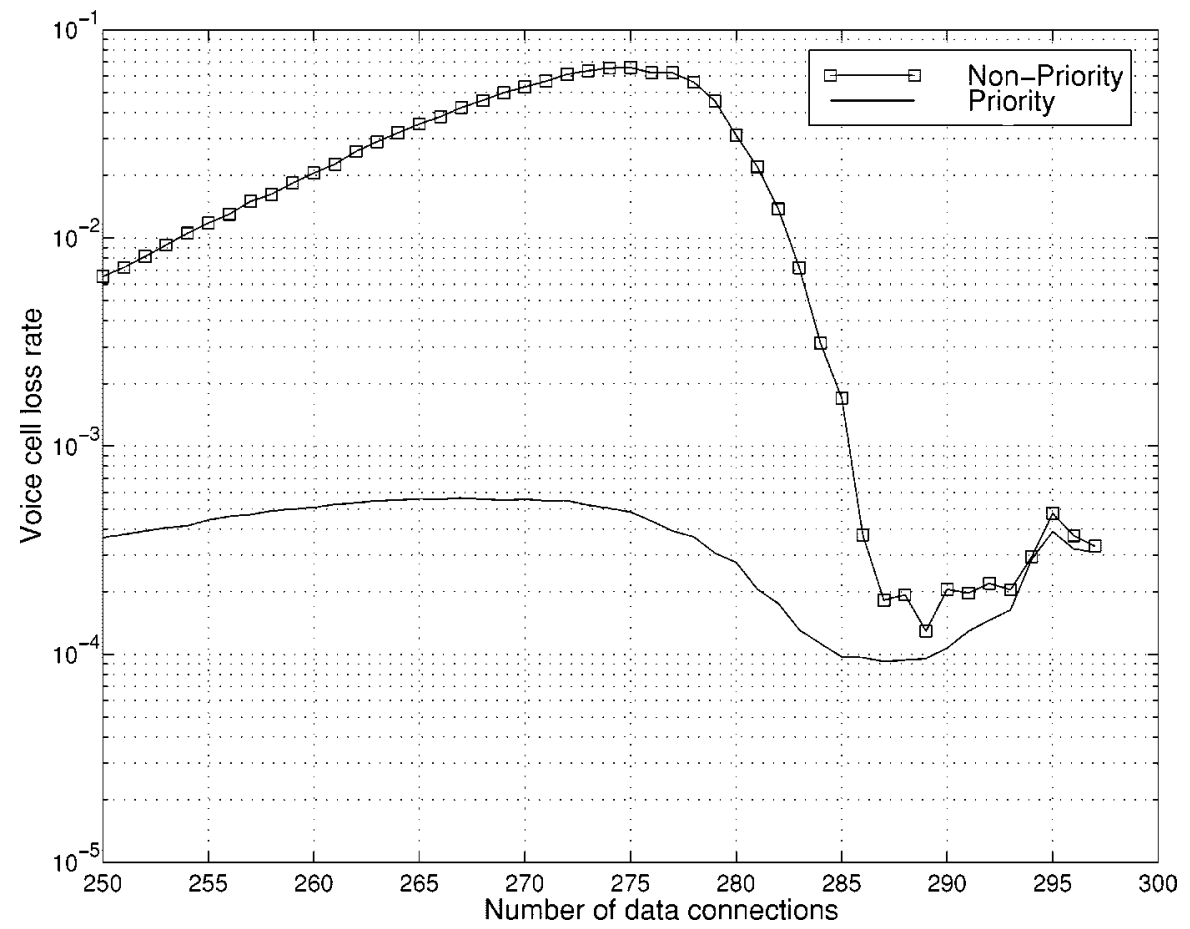

Fig. 7. Voice loss rate as a function of the number of data connections with 100 voice connections.

creases further, the allocation algorithm can maintain a reasonable data traffic delay below $100 \mathrm{~ms}$ up to a throughput of $96 \%$ (with 14 VBR connections). For higher offered loads, the data QoS rapidly decreases.

\section{B. Performance with Voice and Data Traffic}

Another set of simulations have been run with only voice and data connections to illustrate the specific impact of the FPBP protocol on the QoS offered to voice connections. The cell loss rate results presented in Fig. 7 show that, whereas in the nonpriority case (when voice and data control packets are assigned the same priority in the FPBP protocol) the cell loss rate would become high enough to degrade the voice performance as data load increases, the FPBP protocol keeps the voice cell loss rate to an acceptable level such that it is not a limiting factor of the DR-TDMA protocol performance. Therefore, if the number of voice connections is such that the voice cell loss rate is acceptable, any number of data connections can be admitted without 


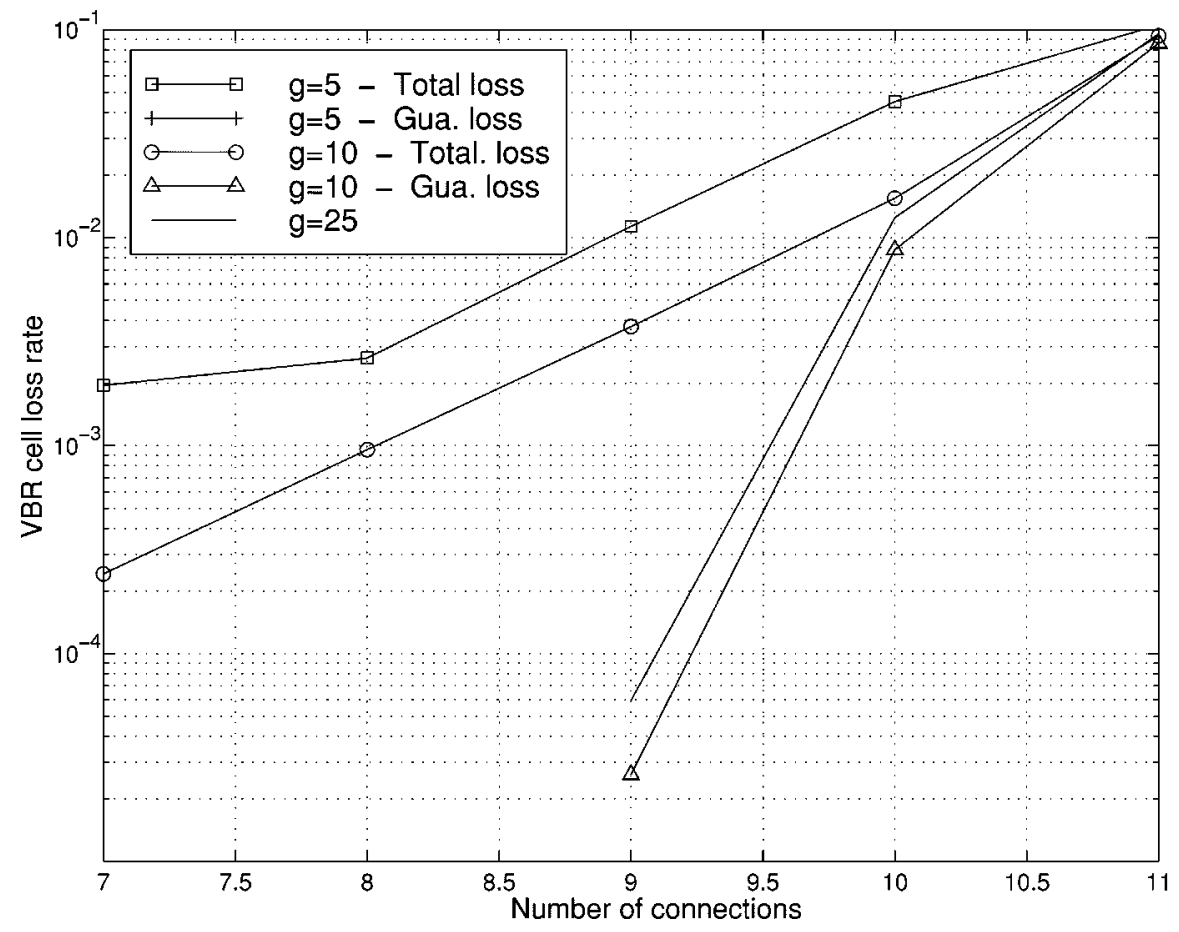

Fig. 8. VBR cell loss rate as a function of the number of VBR connections \#2.

degrading the voice QoS. The decrease in the voice cell loss rate beyond a certain data load is due to the same reason explained in Section V-A (i.e., an increasing number of data requests are piggybacked on data packets, resulting in a reduction of the overall contention traffic and hence a decrease in the voice cell loss rate). Furthermore, the voice cell loss rate performance improvement due to the FPBP protocol does not affect the data delay, and a throughput of $96.5 \%$ can be sustained while keeping the data delay below $250 \mathrm{~ms}$ and a voice cell loss rate lower than $1 \%$ [19].

\section{Performance with VBR Traffic}

Finally, we have simulated the DR-TDMA protocol with VBR connections only to evaluate the performance of the novel VBR resource allocation algorithm that we have presented in this paper. Figs. 8-10, respectively, show the VBR cell loss rate, delay, and throughput as a function of the number of VBR connections; $667 \mathrm{~kb} / \mathrm{s}$ VBR connections (i.e., VBR connections \#2) with the parameters in Table IV were used for this set of simulations. We can see that the total cell loss rate remains low (below 1\%) for an offered load below $90 \%$ to $97 \%$ depending on the guaranteed traffic parameter $g$. We observe that when the parameter $g$ is decreased, the total loss rate increases slightly but, as expected, there is a significant improvement of the loss rate of guaranteed cells. It should be noted that for $g=5$, no loss of guaranteed cells has been observed, hence the lack of a corresponding curve in Fig. 8. These results confirm that our protocol is able to deliver a better QoS to guaranteed traffic.

Another useful performance measure introduced in [9] is the allocation efficiency $(A E)$ defined as the fraction of slots allocated to VBR traffic that are actually used for transmissions. A high $A E$ index indicates a good match between the virtual status at the base station scheduler and the real status of the respective mobile station. Furthermore, a higher $A E$ will allow the system to support more data traffic. For the simulated conditions we have obtained $A E$ values superior to $99 \%$. However, we have to be careful in the interpretation of this number. The allocation efficiency is not the same as the prediction accuracy (i.e., we don't have a $99 \%$ prediction accuracy). Under light traffic conditions, current traffic information can be transmitted to the base station with a low delay. Therefore, prediction of arrivals that occurred before the current frame are mostly accurate. On the other hand, under heavy traffic conditions the prediction will not be as accurate. However, when a slot is allocated to a mobile, it is highly likely that a packet is waiting for transmission. Therefore, the allocated slot is not wasted even if the prediction was not accurate.

For performance comparison purposes, the connection and channel parameters used for the simulations with the VBR connection \#2 have been set similar to those used by Raychaudhuri et al. to evaluate their MAC protocol in [9]. The results presented in Figs. 9 and 10 can thus be compared to the results reported in [9]. At low throughput, both protocols show a stable delay around $2 \mathrm{~ms}$. However, if we take a target transmission delay of $5 \mathrm{~ms}$, the maximum throughput of the protocol in [9] is around 64\%, while DR-TDMA attains a throughput of $83 \%$. Note that in this comparison, our throughput values have been modified from the previous results to take into account the $8 \%$ downlink control traffic overhead considered in [9]. Similarly, if we target a 10-ms delay, the maximum throughput that the protocol in [9] can achieve is 72\%, while our DR-TDMA protocol can reach a throughput in the range of $86 \%-88 \%$ depending on the value of $g$. For the AE performance, our DR-TDMA protocol shows a performance superior to $99.1 \%$, while the protocol in [9] has an AE varying between $75 \%$ and $80 \%$. From these 


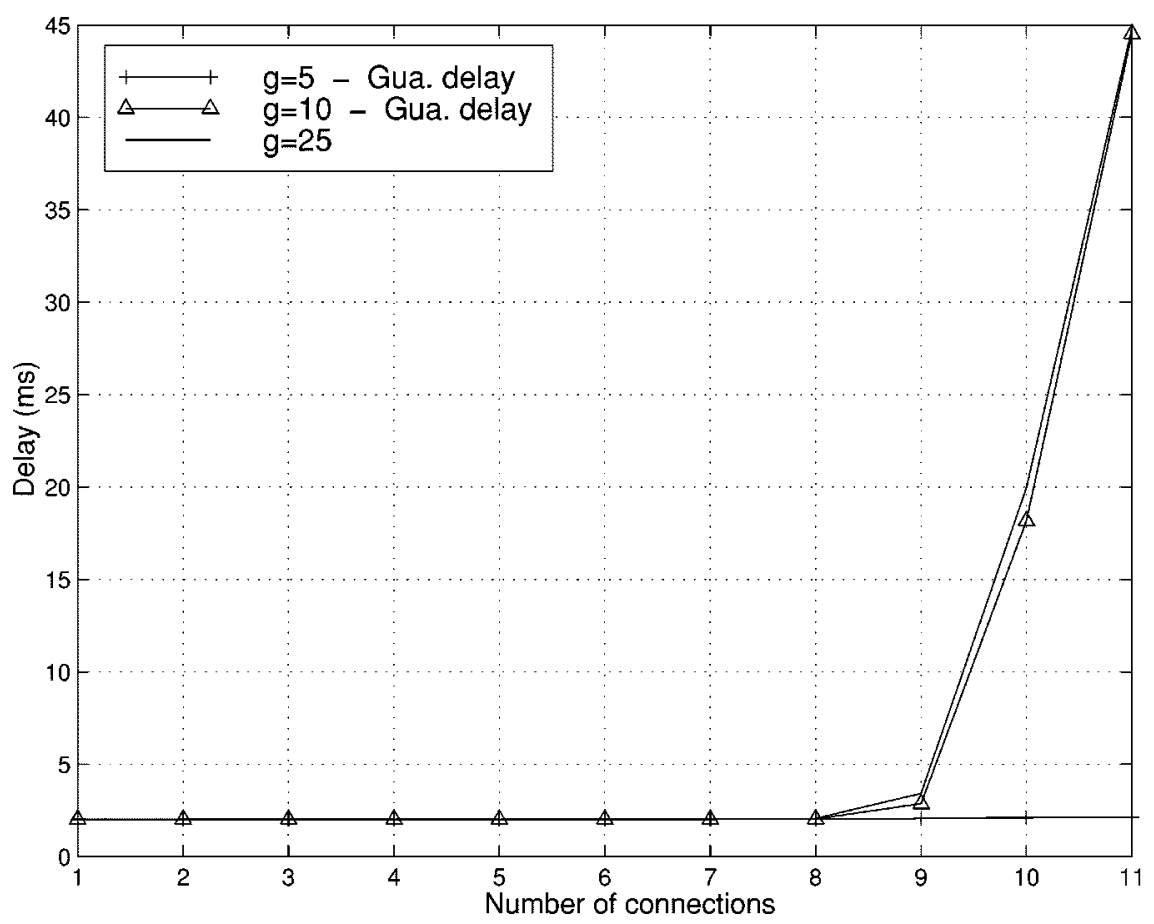

Fig. 9. VBR cell delay as a function of the number of VBR connections \#2.

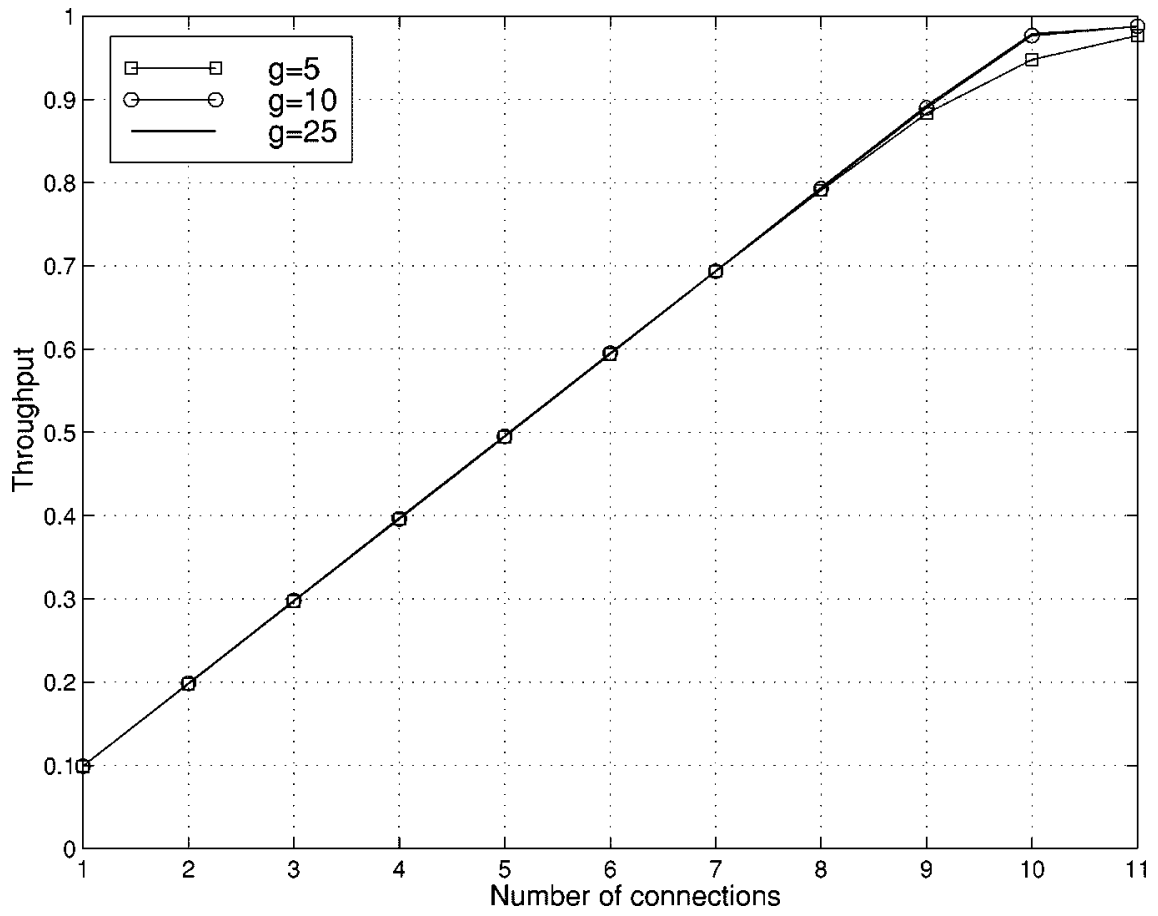

Fig. 10. Throughput as a function of the number of VBR connections \#2.

results, we can see that our DR-TDMA MAC protocol outperforms the bandwidth allocation algorithm proposed in [9] for VBR traffic.

Although both the DR-TDMA protocol and the protocol in [9] use in-band signaling to predict in the scheduler the mobile requirements, they differ in several ways. First, the protocol in [9] has a $15 \%$ fixed overhead for uplink control traffic, while DR-TDMA dynamically adjusts the amount of slots allocated to uplink control traffic as explained in Section IV-D. Therefore,
DR-TDMA can take advantage of periods of low control traffic to increase the bandwidth available to VBR traffic. Second, in [9], only the nature of the buffer length change (increase or decrease) is transmitted from the mobile to the scheduler. This only requires two bits of packet overhead. On the other hand, DR-TDMA transmits the exact change of the instantaneous bit rate. Therefore, the scheduler has a more accurate information of the current status of each mobile station. However, this requires a slightly bigger overhead (approximately 6 bits as es- 


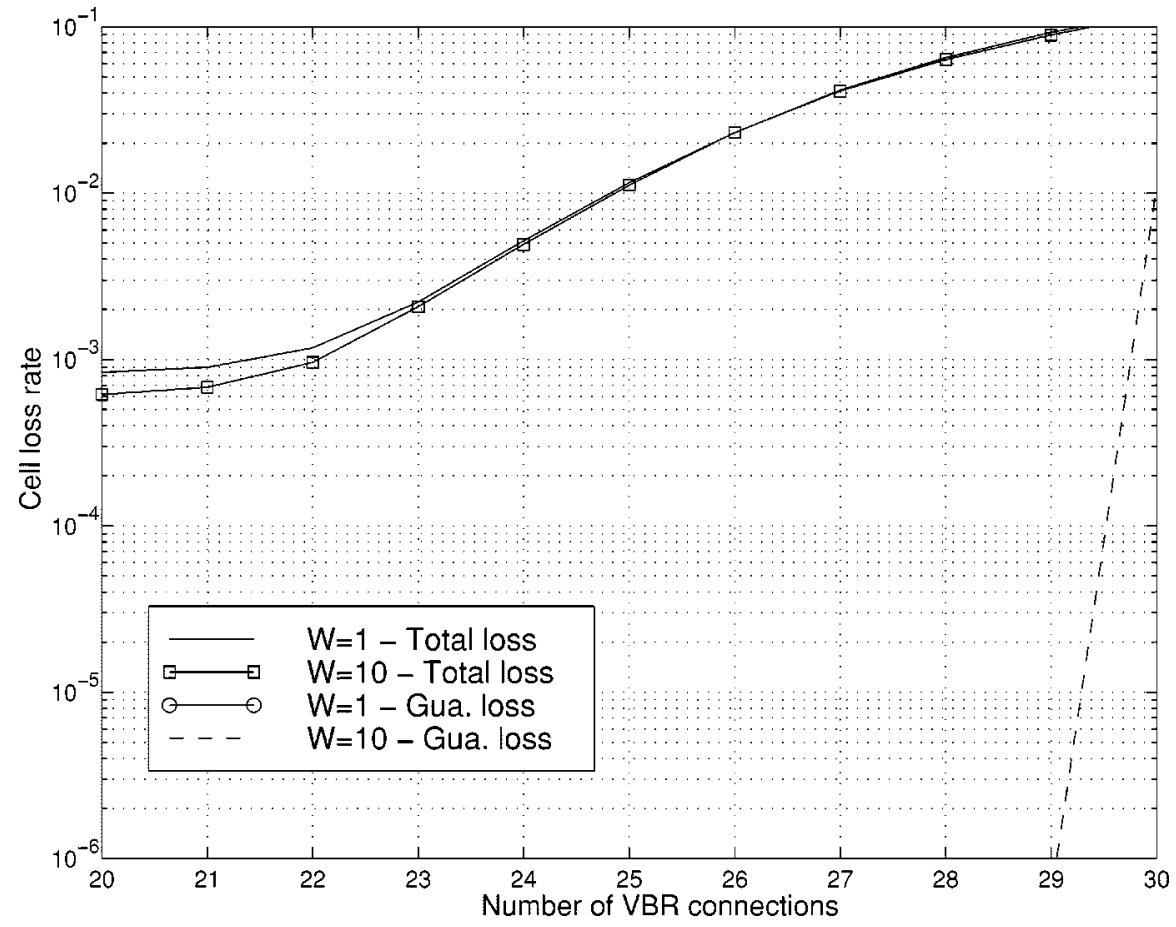

Fig. 11. Cell loss rate as a function of the number of VBR connections \#1.

timated in Section IV-C) which results in a throughput loss of less than $1 \%$ compared to [9]. Also, the VBR source needs to be rate-controlled for our algorithm, while no constraint is imposed by the protocol in [9]. However, this is not a big disadvantage since most of VBR sources are expected to be rate-controlled as explained in Section III-C. Furthermore, our protocol is able to enforce the conformance of the VBR flow with the connection's parameter which is not accomplished in [9].

In the previous simulations, we have used a guaranteed token pool depth $W$ of one token. The parameter $W$ allows a better adjustment of the guaranteed traffic characteristics: when $W$ is set to one, no bursts of guaranteed traffic is allowed, whereas a larger value of $W$ allows for bursts in the guaranteed traffic. We have therefore simulated the DR-TDMA protocol with the parameters of the VBR connection \#1 in Table IV. We have used a sustainable guaranteed cell rate parameter $g$ of 5 to compare the cell control algorithm with token pool depth $W$ of 1 and 10 tokens. Fig. 11 shows the cell loss rate performance for these two values of token pool depth. With $W=10$, the guaranteed traffic is higher, therefore the total cell loss rate is slightly improved while the guaranteed cell loss rate increases similarly to what was observed previously when $g$ was increased. We should note again that for $W=1$, no cell loss has been observed for guaranteed packets and therefore the corresponding curve is missing. Furthermore, for a total cell loss rate of $1 \%$, the offered load of 26 VBR connections \#1 corresponds to a throughput of $92 \%$.

While the impact of the guaranteed token pool depth value on the cell loss rate is small for these traffic conditions, we should recall that the goal of the parameter $W$ is to offer more flexibility on the definition of the guaranteed traffic characteristics. The ON/OFF model used to characterized the VBR sources is known to be slowly varying which explains the small impact of $W$. For burstier traffic, the flexibility that $W$ offers to characterize the maximum burst length can help the user to improve the QoS by better defining the VBR traffic characteristics.

\section{Discussion}

DR-TDMA has a structure similar to previously proposed protocols, especially [3], [8], and [9]. For example, they all use a TDD-TDMA reservation structure. Control packets and in-band signaling are used to transmit partial mobile status information to the base station scheduler to enable prediction of the bandwidth requirements of the sources. However, there are also major differences between DR-TDMA and previously proposed WATM MAC protocols, such as the following:

- dynamic adjustment of the number of uplink control slots;

- contention access priorities provided through the FPBP protocol to improve QoS of real-time connections by expediting the respective control packets;

- use of both minimal in-band and out-of-band signaling to estimate the mobile traffic requirements in the scheduler;

- rate-controlled traffic flow enforced for VBR sources; and

- VBR scheduling offering high QoS to conforming traffic. These differences allow DR-TDMA to offer a higher throughput while maintaining the required QoS.

On the other hand, DR-TDMA has some disadvantages compared to other WATM MAC protocols. First, the proposed allocation algorithm may require more memory and processing power in the base station scheduler. However, this problem is being minimized by continued cost reductions and speed increases in memory and processors. Also, although DR-TDMA requires more overhead bits in data packet for in-band signaling purposes, we have shown in Section IV-C that the overhead can be reduced to 6 bits. Although this overhead has not been reflected in the results previously presented, the 6 overhead bits per WATM cells reduce the net data slot utilization by only 
$1.25 \%$, which is quite small compared to the throughput improvements that result from providing more accurate status information to the base station using these overhead bits (see Section $\mathrm{V}-\mathrm{C})$.

Alternately, should the structure of the WATM packet not allow for in-band signaling, then the DR-TDMA protocol performance may be reduced, but its main features can still be preserved, provided that the single bit used by each VBR packet to indicate whether the packet is guaranteed or best-effort remains. In this case, all the other in-band signaling information needs to be transmitted in control packets, resulting in increased latency and possibly overallocation of data slots. These factors, together with the increase in control traffic, tend to reduce the system throughput. On the other hand, the increased control traffic could enhance the impact of the control traffic priority mechanism offered by the FPBP protocol.

In the evaluation of the protocol, we have assumed an error-free transmission channel. Although the base station prediction algorithms are sensitive to packets losses, they only affect the short term accuracy of the status predictions. The predicted mobile station status will be correctly updated as new packets are received or corrupted packets are retransmitted.

In this paper, we have proposed a per-connection allocation algorithm. Alternately, data slots may be assigned on a per-mobile basis. This reduces the downlink overhead for allocation announcements, and allows the mobiles to make local decisions about allocations for its connections depending on their actual status. However, this approach is useful mainly for mobile platforms supporting a fairly large number of users and their connections so that some degree of statistical multiplexing can be achieved locally at the mobile. For single user mobile stations with typically a small number of connections, the advantage of this approach could be minimal.

\section{CONCLUSION}

In this paper, we have presented a MAC protocol that efficiently integrates multiple BISDN traffic classes over a wireless ATM link. The motivation behind the development of this protocol was to obtain a high throughput efficiency while respecting the QoS requirements of the ATM traffic classes. We have introduced the application of the FPBP protocol to manage the control slot access so as to improve the QoS of time-sensitive connections. A novel rate-based resource allocation algorithm for VBR traffic as well as a companion cell control algorithm have been proposed to increase the VBR allocation efficiency and to facilitate user specification of the VBR traffic characteristics. Finally, an integrated resource allocation algorithm that provides slot allocation priorities to the different services has been described.

We have presented simulation results that illustrate the performance of the DR-TDMA protocol under a wide variety of traffic conditions. The results indicate that DR-TDMA can provide throughput as high as $96 \%$ while limiting cell loss rates to less than $1 \%$ for both voice and VBR traffic, and data delay to less than $100 \mathrm{~ms}$. We have also shown that the
FPBP protocol can maintain the voice cell loss rate at under $1 \%$ regardless of the data traffic loads, whereas the voice cell loss rate would become unacceptable without contention access priority. We have also presented results to demonstrate the efficiency of the VBR allocation algorithm. A throughput of $90 \%$ with a VBR cell loss rate below $1 \%$ was obtained with a number of $666 \mathrm{~kb} / \mathrm{s}$ VBR connections accessing the uplink using the DR-TDMA protocol.

\section{REFERENCES}

[1] D. Bertsekas and R. Gallager, Data Networks, 2nd ed. Englewood Cliffs, NJ: Prentice-Hall, 1992.

[2] D. Raychaudhuri and N. D. Wilson, "ATM-based transport architecture for multiservices wireless personal communication networks," IEEE J. Select. Areas Commun., vol. 12, pp. 1401-1414, Oct. 1994.

[3] D. Raychaudhuri, L. J. French, R. J. Siracusa, S. K. Biswas, R. Yuan, P. Narasimhan, and C. A. Johnston, "WATMnet: A prototype wireless ATM system for multimedia personal communication," IEEE J. Select. Areas Commun., vol. 15, pp. 83-95, Jan. 1997.

[4] M. J. Karol, Z. Liu, and K. Y. Eng, "Distributed-queueing request update multiple access (DQRUMA) for wireless packet (ATM) networks," in Proc. ICC'95, June 1995, pp. 1224-1231.

[5] F. Bauchot et al., "MASCARA: A MAC protocol for wireless ATM," in Proc. ACTS Mobile Summit, Granada, Spain, Nov. 1996, pp. 17-22.

[6] O. Kubbar and H. T. Mouftah, "Multiple access control protocols for wireless ATM: Problems definition and design objectives," IEEE Commun. Mag., vol. 35, pp. 93-99, Nov. 1997.

[7] J. Sanchez, "A survey of MAC protocols proposed for wireless ATM," IEEE Network, vol. 11, pp. 52-62, Nov. 1997.

[8] H. Xie, P. Narasimhan, R. Yuan, and D. Raychaudhuri, "Data link control protocols for wireless ATM access channels," in Proc. ICUPC'95, Tokyo, Japan, Nov. 1995, pp. 753-757.

[9] S. K. Biswas, D. Reininger, and D. Raychaudhuri, "UPC base bandwidth allocation for VBR video in wireless ATM," presented at the ICC'97, Montréal, PQ, Canada, June 1997.

[10] X. Wu, S. Wu, H. Sun, and L. Li, "Dynamic slot allocation multiple access protocol for wireless ATM networks," presented at the ICC'97, Montréal, PQ, Canada, June 1997.

[11] J. F. Frigon and V. C. M. Leung, "A pseudo-Bayesian Aloha algorithm with mixed priorities," ACM/Baltzer Wireless Networks, vol. 7, pp. 55-63, 2001.

[12] R. L. Rivest, "Network control by Bayesian broadcast," IEEE Trans. Inform. Theory, vol. IT-33, pp. 323-328, May 1987.

[13] S. Nanda, D. J. Goodman, and U. Timor, "Performance of PRMA: A packet voice protocol for cellular systems," IEEE Trans. Veh. Technol., vol. 40, pp. 584-598, Aug. 1991.

[14] H. Heeke, "A traffic-control algorithm for ATM networks," IEEE Trans. Circuits Syst. Video Technol., vol. 3, pp. 182-189, June 1993.

[15] J. Ribas-Corbera and S. Lei, "Rate control in DCT video coding for low-delay communications," IEEE Trans. Circuit Syst. Video Technol., pp. 172-185, Feb. 1999.

[16] K. Joseph and D. Reininger, "Source traffic smoothing and ATM network interfaces for VBR MPEG encoders," in Proc. ICC'95, vol. 3, Seattle, WA, June 1995, pp. 1761-1767.

[17] B. Maglaris, D. Anastassiou, P. Sen, G. Karlsson, and J. D. Robbins, "Performance models of statistical multiplexing in packet video communications," IEEE Trans. Commun., vol. 36, pp. 834-844, July 1988.

[18] The ATM Forum, "The ATM Forum traffic management specification,", vol. 4.0, 1996.

[19] J. F. Frigon, "Dynamic Reservation TDMA Medium Access Control Protocol for Wireless ATM Networks," master's thesis, Univ. British Columbia, Aug. 1998.

[20] J. F. Frigon, H. C. B. Chan, and V. C. M. Leung, "A variable bit rate resource allocation algorithm for wireless ATM," presented at the Globecom'99, Rio de Janeiro, Dec. 1999.

[21] D. J. Goodman, R. A. Valenzuela, K. T. Gayliard, and B. Ramamurthi, "Packet reservation multiple access for local wireless communications," IEEE Trans. Commun., vol. 37, pp. 885-890, Aug. 1989. 


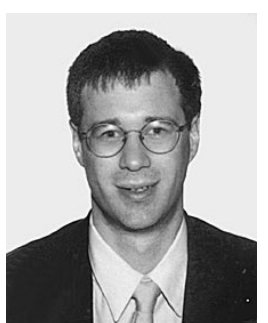

Jean-François Frigon ( $\left.\mathrm{S}^{\prime} 00\right)$ received the B.Eng. degree in electrical engineering from Ecole Polytechnique de Montreal, Montreal, Canada, in 1996, and the M.S. degree in electrical engineering from the University of British Columbia, Vancouver, Canada, in 1998. He is currently pursuing the Ph.D. degree in electrical engineering at the University of California, Los Angeles (UCLA).

In 1998, he was awarded the Governor General's Gold Medal Award as the best graduate student of the University of British Columbia graduating class for the Master's degree. His Master and Ph.D. studies have been partially supported by a postgraduate scholarship from the National Sciences and Engineering Research Council of Canada. His research interests include wireless networks, MAC protocols and design of digital communication systems for high speed wireless communications.

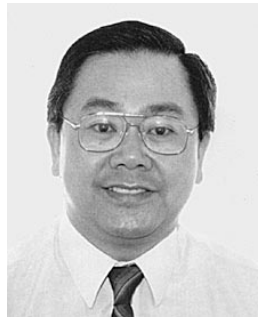

Victor C. M. Leung (S'75-M'81-SM'97) received the B.A.Sc. (Hons.) degree in electrical engineering from the University of British Columbia (UBC) in 1977, and was awarded the APEBC Gold Medal as the head of the graduating class in the Faculty of Applied Science. He attended graduate school at UBC on a Natural Sciences and Engineering Research Council Postgraduate Scholarship and obtained the Ph.D. degree in electrical engineering in 1981.

From 1981 to 1987 , Dr. Leung was a Senior Member of Technical Staff at MPR Teltech Ltd., specializing in the planning, design and analysis of satellite communication systems. He also held a part-time position as Visiting Assistant Professor at Simon Fraser University in 1986 and 1987. In 1988, he was a Lecturer in the Department of Electronics at the Chinese University of Hong Kong. He joined the Department of Electrica and Computer Engineering at UBC in 1989, where he is a Professor and holder of the TELUS Mobility Research Chair in Advanced Telecommunications Engineering. He is also a Member of the UBC Centre for Integrated Computer Systems Research. He is a Project Leader and Member of the Board of Directors in the Canadian Institute for Telecommunications Research, a Network of Centres of Excellence funded by the Canadian Government. He has served on the Editorial Board of the IEEE Journal on Selected Areas in Communications-Wireless Communications Series since 1999. His research interests are in the areas of architectural and protocol design and performance analysis for computer and telecommunication networks, with applications in satellite, mobile, personal communications and high speed networks.

Dr. Leung is a Voting Member of ACM.

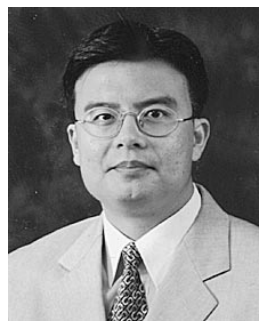

Henry Chun Bun Chan (S'95-M'98) received the B.A. and M.A. degrees from the University of Cambridge, England and his Ph.D. degree from the University of British Columbia (UBC), Canada. He is also studying for a M.B.A. degree by distance learning. He received the Departmental Best Researcher Award in 1999. During his graduate studies, he was awarded the NSERC Scholarship from the Government of Canada, the UBC Graduate Fellowship and the BC TEL Graduate Scholarship. He was the winner of the IEE (HK Centre) Younger Member Paper Contest in 1992.

Between 1988 and 1993, he worked with Hong Kong Telecom primarily on the development of networking services in Hong Kong. From 1989 to 1993, he was also a Visiting Evening Lecturer at the Hong Kong Polytechnic (now known as the Hong Kong Polytechnic University). During his graduate studies, he was a Research and Teaching Assistant in the communication research group of UBC. Between October 1997 and August 1998, he worked with BC Tel Advanced Communications on the development of high-speed networking technologies and ATM-based services. Currently he is an Assistant Professor of the Department of Computing at the Hong Kong Polytechnic University. He is also a Member of the Internet Computing and Electronic Commerce (ICEC) laboratory of the same department. His research interests include Internet technologies, electronic commerce (e-commerce), high-speed networks, wireless networks, mobile computing, and personal communications system. He is writing a book on e-commerce with Prof. Tharam Dillon, Mr. Raymond Lee and Dr. Elizabeth Chang (book proposal accepted by John Wiley \& Sons Ltd., expected to be published in 2001)

Dr. Chan is a Member of ACM and a Member of the Chartered Institute of Marketing. He has served as a Technical Program Committee Member for the high-speed network symposium of IEEE Globecom'99 and as an Organizing Committee Member for APWEB'99. He is currently serving as an Executive Committee Member of the IEEE Hong Kong Section Computer Chapter. 